\title{
Can Classical Roux-N-Y Gastric Bypass Be Combined with Surgical Emergencies? An Interesting Case Series with Literature Review of Two Patients at a Tertiary Care Center
}

\author{
Arnab Mohanty ${ }^{1}$ \\ ${ }^{1}$ Department of General, Minimal Access and GI Surgery, Apollo \\ Spectra Hospital, Gurugram, Haryana, India \\ Int J Recent Surg Med Sci 2022;8:118-122.
}

\begin{abstract}
Address for correspondence Arnab Mohanty, MBBS, DNB Surgery, FMAS (AMASI), Department of General, Minimal Access and GI Surgery, Apollo Spectra Hospital, Sector 8, Gurugram 122001, Haryana, India (e-mail: narcipex@gmail.com).
\end{abstract}

\begin{abstract}
Keywords

- metabolic surgery

- Roux-N-Y gastric bypass

- surgical emergency

Weight-loss surgery is usually recommended to those who walk into our specialist bariatric outpatient department, and intense and often exhaustive counseling, along with a thorough preoperative workup, is undertaken prior to the surgery. All this often fails in the emergent scenario, where the focus shifts to a damage-control mode, and the possibility of a metabolic procedure in this setting, especially one as challenging as gastric bypass, is seldom actively pursued. We discuss two patients presenting to the emergency department with different surgical ailments, but also being obese, with comorbidities, finally undergoing the primary surgeries along with RouX-N-Y gastric bypass, via conventional and laparoscopic methods respectively, with positive immediate outcomes and favorable 1-year excess-body-weight-loss as well. This shows that if patient selection is wise, and adequate expertise is available, gastric bypass can be offered in the emergent scenario as well, with favorable outcomes.
\end{abstract}

\section{Introduction}

Obesity sets the stage for multiple comorbidities and associated systemic illnesses. Bariatric (Metabolic) surgery has progressed rapidly in the last two decades in India ${ }^{1}$ but is still looked upon suspiciously by the population at large, many of whom cannot afford the significant expenses associated with such procedures, and/or do not have medical insurance or access to the necessary information to consider this as a definitive treatment for the disease obesity itself has become.

Many such patients live with obesity, seeking symptomatic care for diseases that spring up as direct sequelae or associations of their morbid habitus. These patients often make their way into the emergency department with surgical ailments that become difficult to diagnose and manage, due to the associated habitus and systemic illnesses.

The traditional approach is to manage the surgical emergency first and then the obesity (if at all), in a sequential manner. But can this be done concurrently? The answer, in a selected group of patients, is logically "yes." Yet, there is not much available literature on how to select such patients, or the procedure of choice. The decision is often left to the discretion of the surgeon and the willingness of the patient. Many patients deny bariatric surgery after recovery, which, needless to say, becomes challenging as well, owing to adhesions and/or altered anatomy resulting from the previous surgery. And when they do agree for a concurrent
DOI https://doi.org/ $10.1055 / \mathrm{s}-0042-1743440$ ISSN 2455-7420.

\footnotetext{
(c) 2022. Medical and Surgical Update Society. All rights reserved. This is an open access article published by Thieme under the terms of the Creative Commons Attribution-NonDerivative-NonCommercial-License, permitting copying and reproduction so long as the original work is given appropriate credit. Contents may not be used for commercial purposes, or adapted, remixed, transformed or built upon. (https://creativecommons.org/ licenses/by-nc-nd/4.0/)

Thieme Medical and Scientific Publishers Pvt. Ltd., A-12, 2nd Floor, Sector 2, Noida-201301 UP, India
} 
procedure, we often see sleeve gastrectomy or an laparoscopic adjustable gastric banding (LAGB) being offered to such patients, in the spirit of damage control.

So, yes, in stable patients with abdominal "urgencies," in whom comorbidities are relatively well controlled, and the field is unlikely to be "significantly" contaminated, we should definitely consider adding a bariatric procedure to the list of surgeries. We discuss two patients (adhering to the CARE [CAse REport] checklist protocols) who presented to the emergency department with different acute surgical conditions, with associated obesity and related comorbidities. Both had been advised metabolic surgery in the past but had deferred due to various reasons. Both patients were screened for surgical risk, counseled thoroughly, and agreed to undergo combined procedures, though via different techniques.

\section{Case I}

A 51-year-old lady (-Fig. 1A) with hypertension, on two antihypertensives, and obstructive sleep apnea (OSA) with a body mass index (BMI) of 41.8 presented to the emergency department with right-sided abdominal pain, associated with nonbilious vomiting and low-grade fever for 2 days. On examination, she was found to be febrile, dehydrated, tachycardic, with maintained blood pressures, with a diffuse right hypochondrium and iliac fossa tenderness, positive Murphy's and McBurney's signs, and only voluntary guarding in the rest of the protuberant abdomen. Investigations revealed moderate leukocytosis, prerenal azotemia, and normal liver functions. She was admitted with a differential diagnosis of acute calculous cholecystitis/appendicitis, to rule out acute pancreatitis/adnexal pathology. An ultrasound abdomen revealed acute calculous cholecystitis with a concurrent acute appendicitis! These findings were confirmed on a contrast-enhanced computed tomography (CT), which additionally revealed an appendicolith at the base of the appendix. Given the short history, she was counseled regarding surgical options, which included laparoscopic versus open cholecystectomy, appendectomy with the addition of a suitable bariatric procedure. The risks and benefits of considering the procedures were explained to her and the family, and an informed consent for surgery as well as
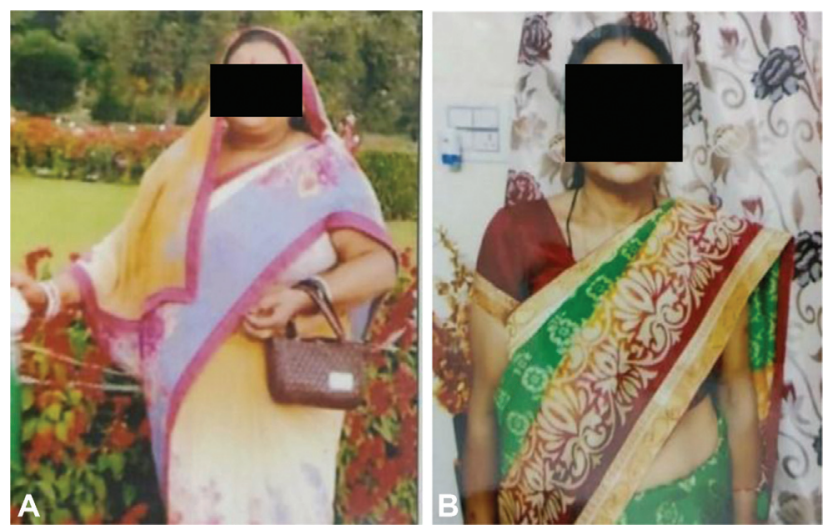

Fig. 1 (A) Case I: 6 months pre-op. (B) Case I: 14 months post-op. publication of outcomes was taken. She opted for conventional open surgery as a matter of choice alone, having been declared fit for laparoscopy after due cardio-respiratory evaluation.

Median celiotomy revealed an edematous gall bladder with multiple calculi and dense omento-cholecystic adhesions, while the appendix (gangrenous up to mid-stalk) had perforated at the tip with a moderate paracecal pus pocket. It was decided to perform an emergency cholecystectomy and appendectomy and proceed with a Roux-N-Y gastric bypass. The procedure was chosen as the patient had a long history of reflux-related symptoms (a relative contraindication for sleeve gastrectomy), though there was no hiatal hernia and endoscopies had suggested adequate medical control with proton pump inhibitors; and inserting a band into a potentially contaminated field was considered against.

The patient went through the entire procedure in 258 minutes, with a 25 cc gastric pouch, end-end anastomosis (EEA) antecolic, antegastric gastrojejunostomy, $150 \mathrm{~cm}$ Roux limb, $70 \mathrm{~cm}$ jejunal limb, and a common channel of $120 \mathrm{~cm}$. She made an uneventful recovery and was allowed oral fluids on postoperative day 2, after a water-soluble contrast study revealed no leakage of contrast. She was discharged on the seventh postoperative day, after suture removal, in a stable condition.

Follow-up in the outpatient department was at 6 weeks, 3 months, and 1 year. At 1 year of follow-up ( $\mathbf{- F i g . ~ 1 B ) , ~ s h e ~}$ had lost $60 \%$ excess body weight, with mild iron deficiency anemia (Hb 10.1). Replacement of iron, vitamin B12, calcium, and other nutrients was chalked out carefully with the dieticians. She had well-controlled blood pressures and had been off antihypertensives for 6 months. Her hemoglobin A1c (HbA1c) was 5.1 and lipid profile had shown considerable improvement on diet alone. Snoring had reduced and the quality of sleep had improved.

\section{Case II}

A 55-year-old gentleman, recently diagnosed with severe OSA, reflux esophagitis, acid peptic disease, BMI of 43.2, bilateral osteoarthritis of knees, uncontrolled hypertension, and diabetes mellitus controlled on long-acting insulin, presented to the emergency department with a colicky pain for a week, over the site of a previously diagnosed incisional hernia following open cholecystectomy 8 years prior. He also complained of periumbilical pain, reddish discoloration, and persistent foul-smelling discharge from the umbilicus for the past year. Examination revealed a morbidly obese gentleman, with tenderness over the Kocher's scar of cholecystectomy, and an ill-defined incisional hernia centered over the scar. The rest of the abdomen was distended, but there was evidence of diffuse peritonitis. The umbilicus was covered with a crust; there was periumbilical erythema and a serous foul-smelling discharge associated with tenderness on pressure. He was admitted with a diagnosis of incarcerated incisional hernia and omphalitis, to rule out urachal remnant. He had normal routine investigations, with an HbA1c of 9, but borderline-deranged sugars. Cardiac 
workup revealed a grade I diastolic dysfunction with early features of pulmonary hypertension. He had severe OSA on sleep study prior to this presentation but had been unwilling for OSA/bariatric surgery. An ultrasound abdomen revealed an incarcerated incisional hernia with enterocele, and a CT confirmed the findings, with no evident bowel compromise, or significant domain shift. The additional finding on CT was that of a urachal cyst, with hyperdense contents. He also had a paraumbilical hernia $(3 \mathrm{~cm}$ defect) with omentocele.

He was counseled extensively and opted for laparoscopic management of the hernia and the urachal cyst, along with a Roux-N-Y gastric bypass (for the same reasons as in the previous patient). Diagnostic laparoscopy in the French position revealed an omentocele in the paraumbilical hernia $(3 \times 3 \mathrm{~cm}$ defect; - Fig. 2). There was a thickened and enlarged urachal remnant with a proximal cyst (-Fig. 3). A $6 \times 7 \mathrm{~cm}$ hernia at the Kocher incision site (-Fig. 2) with dense omental adhesions, and small bowel and transverse colon as content was noted; take down performed using the harmonic scalpel; and bowel found to be congested, but otherwise healthy. Once the larger hernia was taken down, we had access to the hiatus and stomach to proceed with gastric pouch creation. In completion, he underwent laparoscopic hernia reduction, urachal cyst resection, $30 \mathrm{cc}$ gastric pouch side-side gastrojejunostomy using Endo-GIA (gastrointestinal anastomosis) staplers, $150 \mathrm{~cm}$ Roux loop, side-side jejunojejunostomy with a $75 \mathrm{~cm}$ jejunal biliopancreatic limb and $100 \mathrm{~cm}$ common channel, and finally finishing with two IPOMs (intra-peritoneal onlay mesh) $(15 \times 15 \mathrm{~cm}$ for the paraumbilical, and $20 \times 20 \mathrm{~cm}$ for the incisional hernia de-

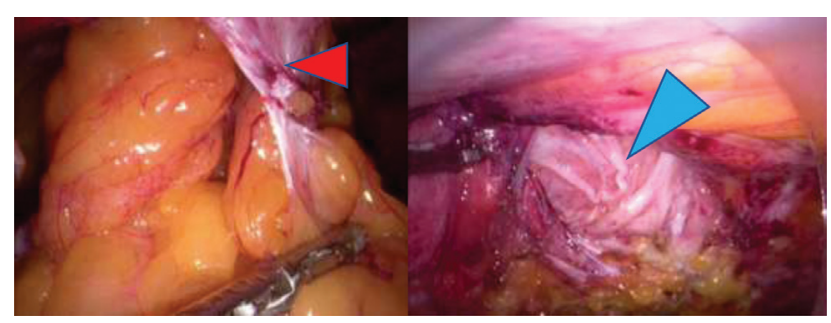

Fig. 2 Case II: Laparoscopic view of the hernial defects. Red arrow, paraumbilical hernia; Blue arrow, Kocher's incision hernia defect.

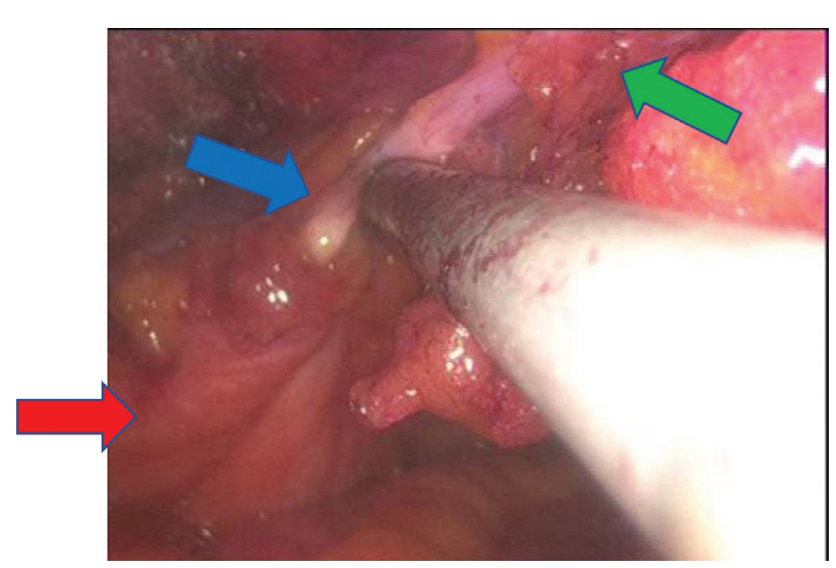

Fig. 3 Case II: Intraoperative view of the pelvis. Blue arrow, urachal remnant; Green arrow, urachal cyst; Red arrow, urinary bladder. fect); the entire procedure lasting 325 minutes. Additionally, an omphalectomy was performed with fashioning of a neoumbilicus. Interestingly, the anesthesiologists struggled to position the Ryle's tube adequately, and the procedure had to be performed without the Ryle's tube being placed in the gastric pouch at all!

He recuperated without systemic complications and was allowed orally in a graded manner, as in the previous patient. He was discharged in a stable condition on the 12th postoperative day, the delay being attributed to an intraoperative propofol extravasation-related arm cellulitis.

He was followed up as in the first case, and by the first year of follow-up had lost $50 \%$ excess body weight, with no detectable deficiencies. Snoring had stopped completely. He was off insulin, with adequate glycemic control and an HbA1c of 6.1 (pre-op was 9.0) on Metformin alone. He was put on regular dietary supplements, as in the previous case, and is on regular follow-up.

\section{Discussion}

The jury is out on what might be the best approach to obese patients presenting with other diagnoses, such as uncomplicated hernia, ${ }^{2}$ and the options range from both simultaneously to bariatric surgery first, and subsequent hernia repair after weight loss or hernia repair followed by bariatric surgery at a later point in time. ${ }^{3}$ But there is no real consensus in the asymptomatic group of patients, let alone in those presenting with complications in the hernia, or other emergent inflammatory or neoplastic diagnoses. ${ }^{4}$ There is also no consensus regarding the hernia size or the extent of domain loss that might preclude combining a bariatric procedure with it. ${ }^{5}$

We found no literature on choice of bariatric procedures in clean-contaminated or contaminated fields; however, we agree that the choice of procedure may be somewhat limited in terms of abstaining from the use of prosthetic devices such as adjustable bands or prosthetic component (layered) meshes, parts or whole of which might get infected. ${ }^{6}$

Sleeve gastrectomy is preferred by many metabolic surgeons in India at the moment, and being technically less demanding, not to mention a relatively fast procedure, has rapidly become the most frequently performed procedure in various countries at present. ${ }^{1,7,8}$ However, one has to consider the clinical profile of patients, their choice and fitness, surgeon experience ${ }^{9}$ and comfort level, the availability of equipment and assistants, possibility of follow-up, compliance of the patient to dietary restrictions and abstinence from addictions, need or possibility of reversal or revision, and the financial status of the paying patient, while deciding on the ideal procedure for that patient in any particular situation. ${ }^{10}$ The best postoperative control of comorbidities has arguably been achieved with the Roux-N-Y gastric bypass, irrespective of the technique, ${ }^{11-13}$ and this leads to many patients choosing this procedure after counseling, and us in promoting this procedure as a definitive, though irreversible, procedure for loss of excess body fat as well as control of comorbid status. Having said that, one must 
remember that literature is evolving continually, and new evidence points that there may not be much to choose between the two procedures ${ }^{14}$ after all. Hence, the procedure of choice is a matter of much conjecture, and highly dependent on the surgeon and/or patient, and, at times, one that is mutually agreeable to.

Both our patients were discharged within an acceptable time frame, without any surgery-related acute complications, and both achieved appropriate long-term goals as well. However, since the concept of combining extensive metabolic procedures with emergency surgeries is not routinely followed practice yet, we do not currently possess literature regarding the probability of success or failure when applied to a larger population. The possible complications of such combinations would be related to treatment of either the acute surgical illness, the metabolic procedure, or both, and also to the overall morbidity of the patient as well. Commonly anticipated complications would be anastomotic leaks, mesh infection, early recurrence of hernia, peritonitis and sepsis in the early postoperative period, and failure to achieve target weight loss/comorbidity control or secondary herniation (external or internal) in the long run. Though further powered and controlled studies are needed to reach statistical figures regarding practicality of such combinations, it is certain that an individualized approach will always be needed to determine the feasibility and choice of procedure in each patient being counseled toward such challenging combined surgeries.

\section{Conclusion}

In closure, one can surmise that the concurrent performance of a suitable metabolic procedure should not be refuted blindly, but the procedure of choice and technique (open or laparoscopic) depends on the willingness of the patient and his/her family, their current hemodynamic profile, comorbid status (the American Society of Anaesthesiologists [ASA] criteria should suffice), the urgency and severity of the acute surgical condition, the estimated contamination and alteration of anatomy due to the current disease, availability of equipment, financial implications, and, most importantly, the experience of the surgical team in handling complex surgical situations. This list of variables, exhaustive as it sounds, is not all inclusive, and further controlled and powered trials would shed more light on this conundrum of sorts.

\section{Consent}

Informed written consent has been obtained from both patients, toward the performance of the surgeries and the publication of this series as well, including the masked images, wherever necessary and applicable.

\section{Funding}

No funds were received from any external or internal body toward aids or sponsorship in the conduct of this study.

\section{Conflict of Interest}

I declare that there was no conflict of interest in the performance of this study.

\section{Acknowledgment}

I sincerely thank the patients for their faith in the treatment and their willingness to be part of this series.

\section{References}

1 Bhasker AG, Prasad A, Raj PP, et al; Obesity and Metabolic Surgery Society of India (OSSI) Trends and progress of bariatric and metabolic surgery in India. Updates Surg 2020;72(03):743-749

2 Mathur A, Mathur PK. Is it safe to combine ventral hernia repair with bariatric surgery? Int Surg J. 2020;7(10):3455-3458

3 Olmi S, Uccelli M, Cesana GC, et al. Laparoscopic ventral hernia repair in bariatric patients: the role of defect size and deferred repair. Obes Surg 2020;30(10):3905-3911

4 Sadi S, Sugarbaker PH, Shope T. Case report of combined surgical oncologic and bariatric procedures. Int J Surg Case Rep 2018; 50:5-8

5 Krivan MS, Giorga A, Barreca M, Jain VK, Al-Taan OS. Concomitant ventral hernia repair and bariatric surgery: a retrospective analysis from a UK-based bariatric center. Surg Endosc 2019;33(03): 705-710

6 Venara A, Hubner M, Le Naoures P, Hamel JF, Hamy A, Demartines $\mathrm{N}$. Surgery for incarcerated hernia: short-term outcome with or without mesh. Langenbecks Arch Surg 2014;399(05):571-577

7 Nguyen NT, Nguyen B, Gebhart A, Hohmann S. Changes in the makeup of bariatric surgery: a national increase in use of laparoscopic sleeve gastrectomy. J Am Coll Surg 2013;216(02):252-257

8 Pucci A, Batterham RL. Mechanisms underlying the weight loss effects of RYGB and SG: similar, yet different. J Endocrinol Invest 2019;42(02):117-128

9 Nguyen NT, Masoomi H, Magno CP, Nguyen XM, Laugenour K, Lane J. Trends in use of bariatric surgery, 2003-2008. J Am Coll Surg 2011;213(02):261-266

10 Apovian CM, Huskey KW, Chiodi S, et al. Patient factors associated with undergoing laparoscopic adjustable gastric banding vs Roux-en-Y gastric bypass for weight loss. J Am Coll Surg 2013; 217(06):1118-1125

11 Lutz TA, Bueter M. The physiology underlying Roux-en-Y gastric bypass: a status report. Am J Physiol Regul Integr Comp Physiol 2014;307(11):R1275-R1291

12 Park CW, Torquati A. Physiology of weight loss surgery. Surg Clin North Am 2011;91(06):1149-1161, vii

13 Marti-Fernandez R, Cassinello-Fernandez N, Cuenca-Ramirez $M D$, et al. Roux-en-Y gastric bypass as an effective bariatric revisional surgery after restrictive procedures. Obes Facts 2020; 13(03):367-374

14 Peterli R, Wölnerhanssen BK, Peters T, et al. Effect of laparoscopic sleeve gastrectomy vs laparoscopic Roux-en-Y gastric bypass on weight loss in patients with morbid obesity: the SM-BOSS randomized clinical trial. JAMA 2018;319(03):255-265 
